DOI: $10.25100 /$ pfilosofica.v0i51.10242

\title{
HEIDEGGER EN ACCIÓN. APROXIMACIONES A LA PERSPECTIVA PRÁCTICA DEL PENSAMIENTO EN DOS TRAMOS DE SU OBRA
}

\author{
Luis Fernando Butierrez \\ Universidad Nacional de La Plata, Buenos Aires, Argentina.
}

\begin{abstract}
Resumen
En este trabajo nos proponemos indagar en la modalidad práctica del pensamiento de Heidegger a partir de sus consideraciones en torno a la praxis y la acción, en trabajos fundamentales de la época de Sein und Zeit en comparación con elaboraciones de su periodo posterior a la Kehre. En este marco, buscaremos demostrar cierta continuidad entre ambos períodos a partir de sus consideraciones ontológicas de la praxis, lo cual permite interpretar el carácter correlativo del pensar y la acción. Para dar cuenta de ello, en primer lugar, abordaremos su respectiva interpretación de la tradición aristotélica en paralelo con las elaboraciones de la analítica en 1927. En segundo lugar, analizaremos un conjunto de consideraciones prácticas del pensar en trabajos posteriores a 1930. De esta manera, esperamos especificar la modalidad práctica del pensamiento de Heidegger, tanto en sus elaboraciones como en su propia articulación.
\end{abstract}

Palabras clave: pensamiento; praxis; mundo; ontología; filosofia.

Cómo citar este artículo: Butierrez, L. F. (2020). Heidegger en acción. Aproximaciones a la perspectiva práctica del pensamiento en dos tramos de su obra. Praxis Filosófica, (51), 77-96. doi: 10.25100/ pfilosofica.v0i51.10242

Recibido: 23 de enero de 2020. Aprobado: 5 de marzo de 2020. 


\title{
Heidegger in Action. Approaches to his Practical Perspective of Thought in Two Sections of his Work
}

\author{
Luis Fernando Butierrez ${ }^{1}$
}

\begin{abstract}
In this work we propose to investigate the practical modality of Heidegger's thinking based on his considerations about praxis and action, in fundamental works of the time of Sein und Zeit compared to elaborations of his postKehre period. In this framework, we will seek to demonstrate some continuity between the two periods based on their ontological considerations of praxis, which allows us to interpret the correlative nature of thinking and action. To account for this, first, we will address their respective interpretation of the Aristotelian tradition in parallel with the elaborations of analytics in 1927. Second, we will analyze a set of practical considerations of thinking of works after 1930. In this way, we hope to specify the practical modality of Heidegger's thinking, both in his elaborations and in his own articulation.
\end{abstract}

Keywords: Thought; Praxis; World; Ontology; Philosophy.

${ }^{1}$ Doctor y Profesor en Filosofía (UNLP); Maestrando en Clínica psicoanalítica (Universidad Nacional General San Martin). Docente e investigador en filosofía contemporánea (UNLP) y filosofía del lenguaje (UCES). Sus áreas de trabajo e investigación son: pensamiento, lenguaje, subjetividad y alteridad en el pensamiento de Heidegger, Derrida y Lacan (Filosofía Contemporánea y Filosofía del Lenguaje).

ORCID: 0000-0001-9502-2234 E-mail: luisbutierrez@yahoo.com.ar 


\title{
HEIDEGGER EN ACCIÓN. APROXIMACIONES A LA PERSPECTIVA PRÁCTICA DEL PENSAMIENTO EN DOS TRAMOS DE SU OBRA
}

\author{
Luis Fernando Butierrez \\ Universidad Nacional de La Plata, Buenos Aires, Argentina.
}

En los últimos años, el amplio espectro de estudios académicos de la obra de Heidegger tiene entre sus tópicos recurrentes la consideración de la praxis y el pensamiento, tanto en sus elaboraciones teóricas, como en la especificidad biográfico-histórica de su propia posición como pensador contemporáneo. Estos estudios pueden agruparse, al menos, desde dos enfoques.

Por un lado, desde la recepción heideggeriana de la praxis en Aristóteles, a partir de las ya clásicas lecturas de Volpi ${ }^{2}$. En esta línea, los análisis en torno a sus elaboraciones de Sein und Zeit (SuZ) se desarrollan en paralelo con aquellos centrados en el mundo de la técnica y el pensar en el horizonte del Ereignis, en sus elaboraciones después de la Kehre, desde 1930. Así planteada la distinción, puede articularse la perspectiva heideggeriana de la praxis con su concepción del pensamiento. En este sentido, las dinámicas del pensar que distingue en su tentativa de torsión de la metafísica en sus trabajos posteriores, permiten reconocer modalidades prácticas del pensar que bien pueden contribuir con el desarrollo de los estudios en torno a la praxis de sus trabajos de los años 20 .

En este contexto, se encuentra abierto el debate sobre continuidades o discontinuidades del tratamiento de la praxis en el período de madurez de Heidegger, en especial, desde las posiciones críticas que distinguen

\footnotetext{
${ }^{2}$ Véase Volpi, 1994; 1996; 2007; 2012, entre otros.
} 
un abandono explícito de tales consideraciones, en consonancia con su programa orientado al despliegue del pensamiento del ser, entendido como una modalidad activa del pensar marcadamente separada del actuar ${ }^{3}$. Frente a ello, encontramos interpretaciones que distinguen un abandono de la equivalencia phronesis-Sorge en SuZ y su carga subjetivista, para desarrollar relaciones de continuidad en el par phronesis-Ereignis, desde sus elaboraciones posteriores ${ }^{4}$.

Por otro lado, podemos reconocer un enfoque de las cuestiones de la acción y el pensamiento en la obra de Heidegger orientado a debates de orden ético y político. En este sentido, destacamos las discusiones en torno a la ausencia o presencia de una propuesta ética en sus trabajos o de una orientación afín en su labor filosófica ${ }^{5}$. Ello se encuentra imbricado no solo con las cuestiones generales en torno a su modalidad de pensamiento, sino también con las consideraciones políticas de su perspectiva y su posición histórica en la época del Nazismo. En este marco subrayamos las interpretaciones que destacan, en línea arendtiana, el sesgo especulativo y supremacista del pensamiento de Heidegger, articulado en una despolitización de la praxis y consecuente retirada de los asuntos públicos y del mundo ${ }^{6}$.

En estos enfoques y discusiones se actualiza la necesidad de lograr una aclaración suficiente del estatuto del pensamiento en la obra de Heidegger, en el marco de las variaciones de su teoría de la praxis o de la acción. El presente ensayo se inscribe en estas coordenadas, por la vía de una indagación en torno a la modalidad práctica de su pensamiento en diferentes momentos de su obra. Entendemos que un desarrollo respectivo puede establecer las condiciones para articular algunas de las preguntas implicadas en aquellas recepciones y lecturas de la obra heideggeriana: ¿puede distinguirse una teoría de la praxis en los dos períodos fundamentales de su obra?; ¿es legítimo el cuestionamiento y la indagación de un programa ético en y desde sus propias elaboraciones? ¿es posible evaluar el propio despliegue del pensamiento y posición histórica de Heidegger a partir de su propio marco categorial y teórico?, entre otras.

Nuestra hipótesis consiste en afirmar que es posible sostener cierta continuidad entre las respectivas elaboraciones de la época de SuZ y de los trabajos posteriores a la Kehre, a partir del esclarecimiento del ámbito ontológico primario y anterior a toda acción histórica determinada. En

\footnotetext{
${ }^{3}$ Véase Carosio, 2014, pp.16, 23; Calle Zapata, 2016, p. 59.

${ }^{4}$ Véase Adrián, 2010, pp. 284-290; Thurnher, 1991, p. 13.

${ }^{5}$ Véase Cerezo, 1991, p.73; Calle Zapata, 2016, p. 60; Muñoz, 2010, pp. 99-108, entre

${ }^{6}$ Véase Di Pego, 2019a, pp. 213-219; Di Pego, 2019b, p. 204; De Lara, 2014, p. 78.
} otros. 
este sentido, entendemos que la modalidad de pensamiento que Heidegger desarrolla y articula a lo largo de varios tramos de su obra se haya imbricado con un concepto originario de praxis, que permite interpretar el carácter correlativo e irreductible del pensar y la acción.

Para dar cuenta de ello, en primer lugar, retomaremos aspectos fundamentales de la recepción heideggeriana de la praxis en Aristóteles, en sus trabajos de principio de los años 20 y en SuZ, buscando poner de manifiesto la modalidad del pensar que allí articula. Luego, analizaremos un conjunto de aspectos prácticos del pensar que Heidegger distingue en tramos de sus trabajos del período posterior a la Kehre. Por medio de un conjunto de notas, intentaremos no descuidar cuestiones internas a su obra en tensión con nuestra hipótesis de continuidad, así como también sugerir algunas discusiones con otras lecturas e interpretaciones respectivas. Finalmente, articularemos los resultados de estas aproximaciones y abordajes, para cotejar en qué medida es posible plantear allí una continuidad y una especificación de la modalidad práctica del pensamiento de Heidegger, en relación a su teoría de la praxis.

\section{Elaboraciones de la praxis y el pensar en camino a Sein und Zeit}

Si abordamos los primeros trabajos de Heidegger en los años 20 podemos reconocer su interés creciente por la orientación de la práctica filosófica hacia la vida y la facticidad ${ }^{7}$. En efecto, desde el conjunto de lecciones impartidas en Friburgo, Heidegger se manifiesta proclive a la búsqueda de una filosofía genuina, no afectada por los prejuicios de la tradición, que inicialmente denomina como ciencia originaria de la vida (Urwissenschaft), orientada hacia una incipiente hermenéutica de la facticidad ${ }^{8}$. En tal enfoque, la filosofía debe ocuparse de la existencia inmediata en el mundo de la vida fáctica ${ }^{9}$. En esta perspectiva que comienza a tomar distancia de las elaboraciones respectivas de Husserl, nuestro autor distingue que el vivenciar se manifiesta rodeado y entramado en significaciones, a partir de

${ }^{7}$ En este marco, consideramos los siguientes trabajos: Zur Bestimmung der Philosophie. 1. Die Idee der Philosophie und das Weltanschauungsproblem (2005) [BP]; Phänomenologische Interpretation ausgewählter Abhandlungen des Aristoteles zu Ontologie und Logik (Anzeige der hermeneutischen Situation) (2002b) [PhIA]; Ontologie. Hermeneutik der Factizität (2008a) [OHF]; Grundbegriffe der aristotelischen Philosophie (2002a) [GbaP]; Platon: Sophistes (1992) [PS] y Sein und Zeit (2000) [SuZ].

${ }^{8}$ Heidegger BP, p.15 [19].

${ }^{9}$ En BP, sus lecciones de 1919, esta tarea lleva el nombre de ciencia originaria de la vida; en PhIA y sus lecciones de 1923, la articula como ontología fenomenológica; en OHF, como hermenéutica de la facticidad, a partir del desarrollo de las estructuras del Dasein sobre dicha base; finalmente, en 1927, esta orientación se consolida en la analítica existencial de SuZ. 
lo cual se propone evidenciar las relaciones entre significatividad y mundo ${ }^{10}$. Para ello, ya en su trabajo denominado Natorp Bericht (1922), subraya la importancia de un abordaje de los conceptos tradicionales para deshacer el estado de interpretación heredado, desde sus encubrimientos y desvíos respecto de las experiencias originarias ${ }^{11}$. Estas modalidades de abordaje, denominadas desmontaje $(A b b a u)^{12}$ y destrucción (Destruktion $)^{13}$, distan de una mera apropiación crítica, pues remiten a un tratamiento práctico de la herencia tradicional, en la búsqueda de conceptos que descubran la estructura originaria de la vida fáctica, como un modo práctico de pensar dicha herencia ${ }^{14}$. En tal sentido, esta modalidad de abordar y leer la tradición filosófica da cuenta del modo específico en que procede el pensamiento de Heidegger, y con ello, nos revelan parte de su modalidad de pensar práctica.

Precisamente aquellas tramas significativas de la vida se revelan con sus interpretaciones de la noción de praxis en la tradición aristotélica, a partir de la distinción entre téchnê y phronesis en la Ética ${ }^{15}$. Desde allí, desarrolla un enfoque de los tipos de acción, confluyendo en lo que Volpi denomina como una ontologización de la praxis ${ }^{16}$. Al respecto, diversos analistas de la obra heideggeriana consideran estas elaboraciones de 1921-22 como fundacionales para la comprensión contemporánea de la praxis humana ${ }^{17}$. En este marco, también suele destacarse la importancia de su seminario de 1924-5, en lo que respecta el desarrollo de aquellas interpretaciones para su consolidación en $\mathrm{SuZ}^{18}$.

En este apartado abordaremos cuestiones fundamentales de estos recorridos del pensar heideggeriano, poniendo atención a sus reelaboraciones respecto de esta perspectiva tradicional, en vistas de su relevancia para la indagación filosófica. En continuidad con los clásicos análisis de Volpi, buscaremos distinguir una fenomenología de la acción técnica, distinguiendo vías de continuidad tanto en su interpretación fenomenológica sobre Aristóteles como en su análisis de la mundaneidad del Dasein en SuZ, en vistas de precisar la modalidad práctica del pensar que articula en ello.

${ }^{10}$ Véase Esguerra Lozada, 2018, pp.159-168.

${ }^{11}$ Véase Butierrez, 2020.

${ }^{12}$ Véase Heidegger OHF, p.75 [99].

${ }^{13}$ Véase Heidegger PhIA, p. 368 [51].

${ }^{14}$ Véase Chiappe, 2012, p. 573.

${ }^{15}$ En especial, desde sus lecturas de los libros VI de Ética a Nicómaco [EN] (1140 a251145 a 10) y IX de Metafísica [M] de Aristóteles desarrollados en: Heidegger PhIAEF:

PhIA; GbaP; PS, entre otros.

${ }^{16}$ Véase Volpi 1996, p. 46.

${ }^{17}$ Véase Gadamer, 2003, p. 76; Volpi, 2012, p. 32. entre otros.

${ }^{18}$ Véase Bernasconi, 1989, p. 130; Thanassas, 2012, p. 43, entre otros. 


\section{I.1 En torno a la tradición aristotélica}

En efecto, en dichos trabajos de los años 20, nuestro autor destaca que la comprensión e interpretación actual del hombre son herederas tanto de la Ética, como de la idea creacionista cristiana ${ }^{19}$. Con el objeto de retomar las experiencias originarias del pensar, desarrolla sus lecturas de la distinción aristotélica entre praxis, phronesis y poiésis, como disposiciones o modalidades de acción, en el marco de la perspectiva del ser del hombre como vida práctica (zoé praktiké) ${ }^{20}$.

Estas lecturas de Heidegger ponen de manifiesto un sesgo práctico de abordaje a partir de la reelaboración y re-jerarquización de tales distinciones, sobre el trasfondo de la búsqueda de ámbitos ontológicos más originarios. Esto le permite subrayar que el trato del Dasein con los entes es derivado de una comprensión ya-abierta en la captación inmediata y unitaria del mundo. En este sentido, entiende que la indagación filosófica debe orientarse por la aclaración de esta relación práctica e inmediata con el mundo, poniendo de manifiesto la experiencia originaria del ser y la comprensión respectiva ${ }^{21}$. De esta manera, nuestro autor busca apartarse del modelo tradicional contemplativo-teorético que entiende la praxis desde la relación objetual con los entes en el mundo. Frente a ello, desarrolla la apertura de un enfoque que revela el carácter de uso y disponibilidad como los modos primarios en que se nos dan los entes ${ }^{22}$. En este sentido, la filosofía entendida como práctica del pensar remite a la actividad y experiencia humana, para su preguntar y despliegue respectivo.

Más en detalle, Heidegger interpreta aquellas disposiciones particulares aristotélicas en el marco de su indagación en torno a la unidad ontológicaestructural del Dasein. Así, frente las cinco determinaciones del alma que Aristóteles distingue para el acceso o la apertura a la verdad ${ }^{23}$, Heidegger delimita dos formas: el ámbito práctico (logistikón) (relacionado con la téchnê y a la phronesis) y el teórico (epistemonikón) (relacionado con la episteme y la sophia) $)^{24}$.

Esta distinción le permite reelaborar la jerarquización aristotélica, a partir del reconocimiento de ámbitos originarios y derivados. Específicamente, en el marco de las respectivas formas de conocimiento distinguidas por Aristóteles (episteme/sophia para la theoria; téchnê para la poiésis y

\footnotetext{
${ }^{19}$ Véase Heidegger PhIA, pp.367-72[50-6].

${ }^{20}$ Véase Aristóteles EN, VI, 5, 1140 a.

${ }^{21}$ Véase Heidegger PhIA, p. 398

${ }^{22}$ Véase Heidegger GbaP, p. 25.

${ }^{23}$ Estas son: epistéme, sophía, téchnê, phrónesis y nous (Aristóteles EN, VI, 3, 1139 b).

${ }^{24}$ Véase Heidegger PS, pp. 28-31.
} 
phronesis para la praxis), Heidegger se contrapone a la consideración eminente de la theoría por su vinculación con la contemplación de lo divino y lo eterno, cuyo carácter necesario lo distingue de los asuntos humanos ${ }^{25}$. Tanto en el Bericht, como en los específicos desarrollos en su curso de 1924-5, nuestro autor subraya que Aristóteles aborda tales cuestiones y sus consideraciones de la phronesis desde la perspectiva de la movilidad (kinesis), como un enfoque apropiado a la contingencia de las acciones y disposiciones del hombre ${ }^{26}$.

Pero nuestro autor subraya que tales elaboraciones no se han constituido desde el ámbito fenomenal propio de la existencia humana, lo cual implica un tratamiento inadecuado del mundo humano de la praxis. En su enfoque, ello se debe a que Aristóteles no logró distinguir la constitución fundamental del Dasein y su unidad ontológica como temporalidad originaria, en parte debido a sus compromisos con la tradición metafísica y su comprensión del ser respectiva ${ }^{27}$.

Precisamente, Heidegger contrapone a dicha comprensión el carácter derivado de la theoría, en tanto modalidad de praxis secundaria respecto al trato práctico con los entes en la cotidianidad del mundo. Ello implica un cambio de acento fundamental: a diferencia del ámbito de lo eterno, la vida humana se caracteriza por un dinamismo, contingencia y finitud que implica un modo propio de acceso ${ }^{28}$. En tal sentido, la teoría es una derivación de nuestra experiencia originaria en el mundo circundante.

Del mismo modo, la praxis, en su carácter originario, articula las formas de acción determinadas, perdiendo con ello la determinación estática atribuida por la tradición contemplativa ${ }^{29}$ : las relaciones cotidianas con los entes remiten, en definitiva, a las posibilidades de acción del Dasein, entendidas como modalidades de despliegue del ser-posible. Esta rejerarquización ontológica de Heidegger implica también el desplazamiento del significado del ser, desde aquella orientación aristotélica a la producción hacia la consideración del uso en el contexto instrumental del Dasein como ser-en-el-mundo ${ }^{30}$.

A partir de estos desplazamientos e interpretaciones, Volpi inscribe la ontologización heideggeriana de la praxis, la cual se aparta de la tradicional

${ }^{25}$ Véase Heidegger PhIA, 388-90[74-6]; Aristóteles M, I, 980b-983a25 y Aristóteles EN, X, 8, 1178a10-b23.

${ }^{26}$ Véase Heidegger PS, pp.48-56; Aristóteles EN, VI, 5.

${ }^{27}$ Véase Taminiaux, 1989, p. 185.

${ }^{28}$ Véase Heidegger PhIA, p.376 [60].

${ }^{29} \mathrm{Al}$ respecto, véase Gethmann, 1993, p. 144; Gethmann, 2007, pp.171- 184.

${ }^{30}$ Véase Heidegger PhIA, p.373 [57]. 
remisión al ámbito ético, para abrirse como modo esencial del ser del Dasein en la inmediatez de la vida fáctica ${ }^{31}$. Desde este enfoque, el mundo deja ya de comprenderse como conjunto objetivo de entes, para abrirse como un ámbito donde se despliegan las posibilidades prácticas del obrar, cuyas remisiones remiten en última instancia al poder-ser o proyecto del Dasein. Con ello, la vida se comprende irreductiblemente unida al mundo, sin una pretensión de exterioridad contemplativa y pura respecto de la experiencia.

Sin embargo, subrayamos que aquella proyección del obrar, más que a un comportamiento (Verhalten) efectivo, remite a la posibilidad de ser del Dasein que se articula precisamente con el obrar determinado. Ello supone un desplazamiento de la comprensión tradicional solo restringida a lo presente, lo cual tiene sus desarrollos de continuidad en las elaboraciones de SuZ.

\section{I.2 La mundaneidad del Dasein en 1927}

En efecto, aquellas interpretaciones se articulan en la perspectiva sobre la praxis que puede distinguirse en los parágrafos 14-19 de SuZ, a partir del desarrollo de la mundaneidad del Dasein, en el marco de la denominada dimensión práxica de la utilizabilidad ${ }^{32}$. Allí, Heidegger parte de los modos en que los entes se nos abren en el mundo circundante: el uso (Gebrauchs) (junto a la utilidad -Dienlichkeit-) y el trato (Umgang). En este contexto, analiza la modalidad primaria de praxis articulada en la estructura ontológica del cuidado (Sorge). Desde esta estructura originaria, Heidegger distingue la ocupación o trato cotidiano (Besorgen), como modalidad específica en que se nos dan los entes en el mundo. Con ello, pone el acento en la apertura del mundo a partir de la relación ontológica entre praxis y cuidado ${ }^{33}$.

Este carácter primario del uso se articula con la distinción de los modos de ser que Heidegger vincula con sus anteriores elaboraciones en torno a la herencia aristotélica. En efecto, la ontologización de la episteme, téchnê y phronesis ${ }^{34}$ aquí se profundiza al ser equiparadas con los modos de ser del carácter de simple-estar-ante (Vorhandenheit), del estar a la mano/ utilizabilidad (Zuhandenheit) y el cuidado, respectivamente ${ }^{35}$. Ello le permite insistir en sus comprensiones respecto de la theoría: cuando el Dasein se

\footnotetext{
${ }^{31}$ Véase Volpi, 1994, p. 332.

${ }^{32}$ Véase Ihde, 1979, p.38.

${ }^{33}$ Véase Heidegger SuZ, pp.67-72[94-9]; Heidegger OHF, pp.101-4[128-32]; Heidegger GbaP, p. 352.

${ }^{34}$ Heidegger traduce phronesis por circunspección (Umsicht). Véase Heidegger PS, p.

47; Heidegger SuZ, pp.67-83[94-109].

${ }^{35}$ Véase Volpi, 2007, p. 36.
} 
dispone teóricamente, el ente se le manifiesta como meramente presente en el mundo, esto es, al modo del simple-estar-ante.

De esta manera, la distinción fundamental entre la presencia y el estar a la mano demarca el ámbito de todo aquello que es en el mundo, al tiempo que permite especificaciones de las relaciones de tales formas de ser con el Dasein. En esta tríada, el trato con los útiles revela la mundaneidad con sus remisiones de significatividad, lo cual ha sido interpretado como el preludio para sus elaboraciones posteriores en torno a la técnica ${ }^{36}$. En tal contexto de pragmaticidad (Zeughaftigkeit), el ente, en tanto útil(Zeug), se revela como ya comprendido en una remisión de significatividad, lo cual pone manifiesto el sentido ya abierto en nuestras relaciones y actividades en el mundo ${ }^{37}$.

Asimismo, el saber o conocimiento que le es propio se manifiesta de modo implícito: es un saber preteorético y constitutivo del mismo movimiento de uso y trato con los útiles y, por ello, no necesariamente explícito o evidente. En este sentido, solo la utilizabilidad del ente permite comprobar este saber de uso ${ }^{38}$. Aquí, nuestro autor destaca las relaciones con el saber hacer o cómo (wie), que se enmarcan en el esto-sirve-para, esto es, en la modalidad de un saber práctico articulado en la estructura de remisión (Verweisung) y de condición respectiva (Bewandtnis) ${ }^{39}$, como conexiones de significatividad y modalidades del para-algo al que se subordinan los diversos tratos con los útiles. Con este enfoque se despliega lo que Gethmann denomina pragmatismo del mundo de la vida: aquél que remite ya no al conocimiento de verdades necesarias, sino a un saber práctico ligado a la acción cotidiana ${ }^{40}$.

En línea con sus elaboraciones de 1922/1925, el trato operativo y técnico en el mundo circundante da cuenta de una finalidad del obrar cuyo empuje no se orienta ya hacia una causa final, sino al tener que ser del Dasein, en su carácter proyectivo. Ello permite poner de manifiesto el modo específico de ser del Dasein en su estructuración unitaria y originaria, no reductible a la mera presencia.

En suma, con estos abordajes y elaboraciones nuestro autor se propone dejar de lado la distinción tradicional entre theoria y praxis, para distinguir esta última como vía de acceso eminente para las relaciones del Dasein en el mundo. En estos trabajos de los años 20, Heidegger subraya que éste es el

\footnotetext{
${ }^{36}$ Véase Cárdenas Arenas, 2005, p.100; Dreyfus, 2006, p. 58.

${ }^{37}$ Véase Heidegger SuZ, p.68 [96]; Rentsch, 1989, p. 92.

${ }^{38}$ Véase Heidegger SuZ, 68s. [96]; Heidegger PS, p. 51.

${ }^{39} \mathrm{El}$ primero, remite al correlato del obrar; el segundo, al Dasein.

${ }^{40}$ Véase Gethmann, 1989, pp.142-6; Chiappe, 2012, p. 568.
} 
ámbito de análisis propio de la filosofía, la cual debe desplegar su preguntar a partir de la experiencia fáctica de la vida, en su propia modalidad temporal.

Junto a ello, las modalidades de interpretación y reelaboración conceptual de tales términos tradicionales nos permiten caracterizar estos procedimientos de Heidegger como formas específicas de articular un pensar práctico, esto es, equiparar su pensar con su tarea filosófica ${ }^{41}$ : un abordaje destructivo de la herencia filosófica que en última instancia remite a las tramas de significatividad y a las comprensiones allí articuladas, en un recorrido hacia experiencias originarias de configuración del sentido y del pensar. A nuestro entender, tales recorridos, modos de abordaje y disposiciones permiten desplegar una dimensión práctica del pensar articulada en el discurso de Heidegger, en continuidad con aquellas elaboraciones en torno a la praxis ${ }^{42}$ : nuestro autor indaga y se desplaza por la herencia del pensamiento y discurso tradicional en relación con las modalidades de ser y comprensión que se articulan en el obrar determinado.

Sin embargo, las elaboraciones de Heidegger en torno a la acción y la praxis no se reducen al abordaje destructivo e interpretación de la tradición aristotélica o a sus elaboraciones de la analítica. La articulación de su propio pensar y discurso hacia 1927 también permite reconocer un conjunto de disposiciones prácticas, especialmente articuladas en SuZ: la co-existencia anticipativa-liberadora y la apertura al llamado de la conciencia para alcanzar la existencia propia, entre otras. En este sentido, el programa de SuZ implica también una tentativa para desarrollar una nueva fundamentación del pensar, en transición respecto a la herencia de la metafísica, que desarrolla en sus elaboraciones posteriores.

\section{Los aspectos prácticos del pensar en elaboraciones después de la Kehre}

A partir de las elaboraciones de Heidegger en los años 30 podemos distinguir la transición hacia un enfoque relacional Dasein-Ser que busca desligarse del lastre conceptual y comprensivo de la metafísica del sujeto. Estos desarrollos se radicalizan en los años 50, en especial, en torno a sus consideraciones del mundo de la técnica, el pensamiento y las posiciones o funciones prácticas del Dasein en su época histórica.

En el contexto de estas cuestiones, retoma la orientación del preguntar y pensar filosófico hacia la clarificación de las esencias de aquello en lo que indaga. Para Heidegger, la esencia (Wesen) remite al ser de aquello que es, lo esencial que hace posible su manifestación, pero no reducido a

\footnotetext{
${ }^{41}$ Véase Betancourt, 2016, pp.21.

${ }^{42}$ Véase Acevedo, 2001, p.7.
} 
sus cualidades presentes, sino abierto a su sentido originario que incluye aquello que se oculta y retrae en la misma manifestación fenoménica ${ }^{43}$. Esta comprensión implica un pensar práctico no reductible a lo mensurable y verificable en la presencialidad del fenómeno, sino a una dimensión que se dinamiza en la propia apertura al $\mathrm{ser}^{44}$, lo cual representa el tono característico del pensamiento de Heidegger ${ }^{45}$.

En el siguiente apartado analizaremos sus especificaciones en torno al pensar y las cuestiones prácticas en el mundo de la técnica, desde un conjunto de trabajos hasta $1960^{46}$. A modo aproximativo, con este recorrido proponemos distinguir la reelaboración práctica del pensar que muestro autor articula después de la Kehre.

\section{1 Perspectivas del pensar}

En los Beiträge de 1938, trabajo de la época considerado fundamental ${ }^{47}$, Heidegger circunscribe dos orientaciones epocales del pensar: a) un primer comienzo (der erste Anfang) de la comprensión del ser, aquella de raigambre griega-metafísica y b) otro comienzo (der andere Anfang) que Heidegger sitúa temporalmente en el por-venir, donde el ser es/será pensado en su verdad como Ereignis, es decir, en una dinámica de co-pertenencia, apertura y donación entre el Dasein y el Ser ${ }^{48}$. En este marco, retomar un camino hacia un pensar no metafísico implica (re)pensar la metafísica en su esencia. Correlativamente, un pensar por fuera de la representación requiere realizarse primero en el mismo marco de la representación, atendiendo las dinámicas y desvíos que con ello se develan. Así, como puesta al límite de esta modalidad, nuestro autor se proyecta en la tarea de un tránsito del pensar de la época. Teniendo en cuenta el carácter ineludiblemente fronterizo y las limitaciones de esta tarea, abandona el programa anterior de una superación de la metafísica (Überwindung der Metaphysik), para proyectarse hacia su torsión (Verwindung) ${ }^{49}$.

${ }^{43}$ Véase Heidegger VA, pp.9-16[9-14].

${ }^{44}$ Véase Inverso, 2019, pp. 218-231.

${ }^{45}$ Véase Agamben, 2016, p. 123.

${ }^{46}$ Aquí consideramos las siguientes obras y entrevistas: Wegmarken (2007b) [WM]; Einführung in die Metaphysik (2003) [EM]; Beiträge zur Philosophie (Vom Ereignis) (2006) [BPE]; Vonträge und Aufsätze (2001) [VA]; Zur Erörterung der Gelassenheit. Aus einem Feldweggespräch über das Denken (1994a) [ZEG]; Brief über den Humanismus (2007a) [BH]; Identität und Differenz (2008b) [ID]; Gelassenheit (1994b) [G] y Spiegel-Gesprächt mit Martin Heidegger (1977) [SGH].

${ }^{47}$ Véase Herrmann FW von ,1994; 1997, passim; Walton, 2012, passim, entre otros

${ }^{48}$ Véase Heidegger BPE, pp. 47-69, 175-91 [54-70; 149-61]; Heidegger EM: 28-30[43s.]; 155-7[183-6].

${ }^{49}$ Véase Heidegger VA, pp. 68-72; 81 [52-4; 62]; Aspiunza, 1995, p. 231. 
En este sentido, también precisa dinámicas apropiadas desde y en el pensar: el salto (Sprung) y el paso atrás (Schritt zurück). Por medio de tales modalidades prácticas busca dirigirse a un pensar lo aún-por-pensar (de aquello ya pensado pero abandonado) ${ }^{50}$. Con el primero, denota un movimiento del pensar articulado en un salto fuera de la metafísica y la representación; con el segundo, refiere a un paso atrás hacia el comienzo de la metafísica. El salto se separa del ser como fundamento (delimitado así por la metafísica), que fue finalmente sustituido por el ente en su mismo modo comprensivo. Es precisamente la comprensión del ser como ente la que conduce a que este pueda ser manejado, manipulado, entendido como algo técnico y bajo nuestro dominio. Teniendo en cuenta esto, el salto no tiene una dirección o ámbito prefijado, sino que el punto de llegada se abre a través del salto.

De esta manera, la dimensión futura del pensar también se desliga de una comprensión ligada a la metafísica de la presencia. En este sentido, estas perspectivas del pensar se tornan fundamentales al momento de contraponerlas a la acción directa, pues su distinción se enmarca en un ámbito de originariedad que es previo al conocimiento y al fáctico estar en el mundo.

En continuidad con sus elaboraciones de los años 20, Heidegger plantea aquí una redefinición de los vínculos entre ser y obrar, en especial, cuando distingue en dicha dinámica transicional una necesidad interna que interpela nuestra esencia y motiva a preparar una región, al modo de un señalamiento (no voluntario) de aquello que aún se mantiene olvidado en el ámbito del primer inicio del pensar ${ }^{51}$.

En este sentido, distingue el pensamiento calculante (das rechnende Denken), caracterizado por una velocidad que no se detiene a meditar, y el pensar meditativo (das besinnliche Nachdenken), el cual requiere un esfuerzo capaz de esperar ${ }^{52}$. Esta modalidad del pensar se articula en un compromiso no dirigido a un querer sino a un esperar sin objeto, en tránsito a la exhortación de aquello por pensar, lo cual permite distinguirlo de la expectativa propia del pensamiento representativo ${ }^{53}$. Esta modalidad disposicional desplaza o dinamiza el pensar, a modo de manifestación última de la voluntad: un compromiso que requiere en un primer tiempo de la voluntad del Dasein (implicada en un dirigirse-templado al ámbito de reserva de su inicio en el pensar) para, en segunda instancia, abandonarla

\footnotetext{
${ }^{50}$ Véase Heidegger ID, pp.40-43; 49s; 58-61[77-81; 95s; 111-117].

${ }^{51}$ Véase Heidegger VA, p.126 [97].

${ }^{52}$ Véase Heidegger G, p. 519 [19].

${ }^{53}$ Véase Heidegger ZEG, p. 43[41]).
} 
desde el temple de la reserva (Verhaltenheit) y la serenidad (Gelassenheit) ${ }^{54}$. En línea con la lectura de Acevedo, destacamos que estos modos de pensar no son excluyentes para Heidegger, sino más bien suplementarios, en el marco de las relaciones dinámicas que destaca para el Dasein ${ }^{55}$.

\section{2 Las posiciones prácticas del Dasein en el mundo de la técnica}

En el marco de esta transición del pensar histórico, la intervención práctica del Dasein es requerida de un modo dinámico y lateral. Por ello Heidegger manifiesta en diversas ocasiones sus críticas a la comprensión del actuar del marxismo ortodoxo, al subrayar que antes que la transformación del mundo es indispensable una transformación del pensar ${ }^{56}$. Dicha dinámica de advenimiento del pensar se abre a partir de disposiciones afectivas fundamentales, correlativas al modo de relación con el mundo circundante, lo cual implica que no puede ser forzado por una conciencia o voluntad.

Sin embargo, esta modalización del pensar es correlativa a un modo de habitar del hombre en el mundo, en su respectiva relación con el ser. Para evitar la carga voluntarista del término resolución (Entschlossenheit/

Entschiedenheit) de la época $S u Z$, en los trabajos de esta época subraya aspectos pasivos y originarios en las dinámicas proyectivas del Dasein, con el objeto de captar su dinámica esencial en una relación de apertura y don, correlativa a un compromiso-entrega con/en tal apertura.

Este cambio de enfoque implica ciertas funciones y relevos relacionales para el Dasein: en cuanto ex-sistente debe guardar la verdad del ser, tal y como Heidegger lo articula en su sintagma: el hombre es el pastor del ser (Hirt des Seins). Esta posición implica un señalamiento práctico de aquello que se retira, en el marco de su función como guardián y vigía ${ }^{57}$. En la misma línea, distingue el rol del Dasein como custodio (Wächter) de la verdad del $\operatorname{ser}^{58}$. En ello, su obrar fundamental consiste en preguntar y pensar de un modo radicalmente opuesto al intervencionismo manipulador del hombre en el marco de la técnica. En este sentido, el impulso para los desplazamientos del pensar proviene del peligro inherente al mundo de la técnica, al modo de un llamamiento o exhortación desde el ámbito de aquello por pensar, hacia la esencia del hombre.

${ }^{54}$ Véase Bulo Vargas, 2008, pp.95.

${ }^{55}$ Véase Acevedo, 2010, p.15.

${ }^{56}$ Heidegger WM, pp. 446 s [362]. En esta línea, sostiene: “(...) la sola acción no cambiará el mundo sin primero interpretarlo (...) es posible que después de siglos el hombre haya actuado demasiado y pensado muy poco. En un mundo que da cada vez más que pensar, el pensamiento no siempre existe" (Towarnicki-Palnier ,1981, p. 6).

${ }^{57}$ Véase Heidegger BH, p.342 [281].

${ }^{58}$ Véase Heidegger BPE, pp. 16s [32]; 99 [92 s]. 
Desde tales distinciones nuestro autor rechaza los planteamientos de un a priori para lo concreto de la existencia fáctica que oriente la práctica, la cual es definible siempre de un modo contingente, en el propio existir y en la época. A lo sumo, sostiene que un pensador tan solo puede esforzarse por construir la pasarela de un tránsito ${ }^{59}$, tal y como se propone con el despliegue práctico de su camino del pensar. Estas especificaciones se inscriben en lo que Feenberg retoma como la diferenciación entre la dimensión óntica y ontológica para la dilucidación de marcos situacionales de la época ${ }^{60}$.

De esta manera, Heidegger insiste en dilucidar los marcos ontológicos y primarios, en este caso, como condición para un obrar y pensar auténtico. En las especificaciones aquí presentadas, el pensar práctico de Heidegger se mantiene así en el ámbito de lo originario, sin reducirse a la presencialidad de los fenómenos, en línea con sus procedimientos de la época de SuZ. No obstante, en su paso hacia la Kehre distingue modalidades prácticas $\mathrm{y}$ orientaciones del pensar que implican roles, disposiciones y funciones específicas, de modo correlativo a su propia posición respeto al pensar metafísico que articula en una tentativa de torsión y desplazamiento. Con ello amplía el alcance de su perspectiva en torno a la labor filosófica, el pensamiento y la praxis desde la época de SuZ, desde un enfoque relacional que busca deshacerse del lastre de la metafísica de la subjetividad.

\section{Consideraciones finales}

Para terminar, proponemos un conjunto de puntualizaciones de nuestro recorrido, con el objeto de circunscribir la perspectiva del pensamiento y el obrar en los tramos considerados de la obra heideggeriana. Desde allí retomaremos nuestras cuestiones iniciales, planteando posibles vías de continuidad en el horizonte de nuestra pregunta por el estatuto práctico y específico del pensamiento de Heidegger.

En primer lugar, retomamos sus elaboraciones e interpretaciones de la praxis aristotélica en sus ya clásicos trabajos entre 1921-1925.En este marco, por un lado, destacamos el horizonte estructural-originario de la indagación de Heidegger: las distinciones en torno a la theoría y la praxis se enmarcan en la búsqueda de estructuras unitarias y primarias de la relación del Dasein en el mundo. Este despliegue del pensar, que busca aclarar el punto de partida fáctico irreductible para la práctica filosófica, se orienta a la tarea de dar cuenta del entramado estructural y originario del Dasein, en el horizonte de la pregunta por el ser. Las limitaciones del lenguaje metafísico heredado y de la perspectiva de la analítica centrada en el Dasein, lo conducen a

${ }^{59}$ Véase Heidegger SGH, p. 683[70].

${ }^{60}$ Véase Feenberg, 2001, p. 447. 
retomar su camino del pensar desde otro enfoque: el pensamiento del ser y las dinámicas relacionales con el Dasein.

La modalidad específica como articula aquel trayecto de su pensar se circunscribe principalmente en la destrucción, desmontaje y reelaboración relacional de los conceptos aristotélicos considerados, en vistas de su programa ontológico y de la aclaración del ámbito apropiado para la indagación filosófica. En este trayecto, destacamos la subversión jerárquica tradicional del pensamiento teórico-contemplativo que nuestro autor interpreta como derivado de una praxis originaria y estructural del Dasein. Es por ello que subrayamos un paralelo entre las disposiciones prácticas del Dasein que especifica en $\mathrm{SuZ}$ y aquella perspectiva de la praxis. En tal sentido, la distinción tradicional pensamiento-acción pierde aquí su sustento para articularse en el marco comprensivo propio de la existencia humana, donde las relaciones prácticas en el mundo no quedan reducidas a lo meramente presente y verificable en acto.

En segundo lugar, abordamos ciertos tramos de la obra de Heidegger entre los años 30 y 50, para dar cuenta de un conjunto de distinciones en torno al pensar y las posiciones prácticas del Dasein, en el marco de sus análisis respecto al mundo de la técnica y la proyección hacia el otro inicio del pensar occidental. A nuestro entender, esta orientación proyectiva insiste en las bases fácticas irreductibles para la indagación filosófica y en la orientación de su práctica hacia ámbitos originarios, con el objeto de recuperar el carácter dinámico, donante y fluido de las experiencias originarias del pensar. Así, en el marco de la facticidad del mundo de la técnica y su pensamiento calculante correlativo, nuestro autor subraya una comprensión no voluntarista o intervencionista de la injerencia práctica del Dasein en su época histórica. En este sentido, la apertura primaria de los temples de ánimo fundamentales, en y desde la experiencia en el mundo, le permite subrayar posiciones anímicamente abiertas e inscriptas en un pensar meditativo, como condiciones de posibilidad de un obrar auténtico que responda a la exhortación de aquél pensar por-venir.

Así, considerando estos tramos de la obra de Heidegger, podemos distinguir una vía de continuidad en sus elaboraciones sobre el pensamiento y la praxis, en especial, a partir de la orientación ontológica de su indagación filosófica. En este sentido, hemos inscripto aquí lo que entendemos como el pensar práctico de Heidegger en sus reelaboraciones, desplazamientos y tratamientos del discurso y pensamiento tradicional. Esta modalidad práctica del pensamiento de Heidegger se articula de manera gradual al modo de una transición respecto del pensar metafísico tradicional y sus bases comprensivas respectivas. En los textos de la Kehre aquí considerados, 
nuestro autor se mantiene en las dilucidaciones del ámbito originario del obrar de un modo correlativo con una modalidad de pensar la tradición metafísica que implican orientaciones, funciones, posiciones, en relación a ella. A nuestro entender, la proyección del pensar en la época de la técnica y las dilucidaciones del entramado anímico-posicional del Dasein, representa una articulación práctica y específica del propio pensar de Heidegger, que aquí desarrolla y extiende su alcance, respecto a la época de SuZ, desplazando o modificando su propia comprensión del alcance de esta tarea que se orienta a una tentativa de torsión de la metafísica heredada. En estas elaboraciones, el carácter anterior, condicional y preparatorio para todo obrar concreto dificulta la viabilidad para aquellas investigaciones sobre un programa ético implícito o de una dimensión óntica del obrar y de la praxis, al menos desde su propio enfoque y marco teórico.

De esta manera, destacamos la relevancia que tienen estas elaboraciones, no solo para una teoría de la acción desligada de las limitaciones tradicionales, sino también para las prácticas contemporáneas mayormente circunscriptas a estos ámbitos anteriores a la acción determinada como el caso del psicoanálisis, por ejemplo.

Aun así, las especificaciones del estatuto práctico del pensar heideggeriano se encuentran todavía por-venir, pues requieren un desarrollo y especificación respectiva en diversos tramos de su obra. En tal sentido, estas continuidades y relaciones también pueden ofrecernos herramientas para pensar y preguntarnos por nuestra propia época técnica y las modalidades de acción que le son correlativas.

\section{Referencias bibliográficas}

Acevedo, J. (2001). Hacia una nueva interpretación del hombre. Heidegger. Cuadernos de Filosofía, (19), 7-19.

Acevedo, J. (2010). La frase de Heidegger "La ciencia no piensa" en el contexto de su meditación sobre la era técnica. Revista de Filosofia, 66, 5 - 23. doi: 10.4067/ S0718-43602010000100001

Adrián, J. (2010). Heidegger y la genealogía de la pregunta por el ser. Barcelona, España: Herder.

Agamben, G. (2016). Lo abierto, el hombre y el animal. Buenos Aires, Argentina: Adriana Hidalgo editora.

Aristóteles. [M]. (1990). Metafisica. (V. García Yebra, trad.). Madrid, España: Gredos.

Aristóteles. [EN]. (1993). Ética Nicomáquea. Ética Eudemia. (J. Pallí Bonet, trad.). Madrid, España: Gredos.

Aspiunza, J. (1995). Heidegger o pensar el futuro. Investigaciones fenomenológicas: Anuario de la Sociedad Española de Fenomenología, (1), 223-236. 
Bernasconi, R. (1989). Heidegger's Destruction of Phronesis. The Southern Journal of Philosophy, XXVIII, Suplemento, 127-147.

Betancourt, W. (2016). El decir del silencio: un homenaje a Martin Heidegger. Praxis Filosófica, (42), 11-32. doi: 10.25100/pfilosofica.v0i42.3165

Bulo Vargas, V. (2008). Verhaltenheit: La tonalidad de un posible nuevo inicio histórico. Revista de Filosofía, 64, 89-98. doi: 10.4067/S071843602008000100007

Butierrez, L. (2020). Tradiciones abiertas. La comprensión de la tradición y las relaciones con la herencia filosófica en dos períodos fundamentales del pensamiento de Heidegger. Revista Tópicos, (en prensa).

Calle Zapata, M. (2016). Martin Heidegger y el intento por pensar la esencia de la técnica como una reorientación en el Ethos. Revista Perseitas, 4(1), 41-61. doi: $10.21501 / 23461780.1803$

Cárdenas Arenas, J. (2005). Filosofía de la tecnología en Martin Heidegger. Praxis Filosófica, (21), 97-110. doi: 10.25100/pfilosofica.v0i21.3233

Carosio, A. (2014). Martin Heidegger: pensar autentico vs progreso técnico. Revista de Filosofia, 78(3), 7-24.

Cerezo Galán, P. (1991). De la existencia ética a la ética originaria. En F. Duque. (Coord.), Heidegger: la voz de tiempos sombríos (pp. 11-79). Barcelona, España: Ediciones del Serbal.

Chiappe, A. (2012). La filosofía de la técnica implícita en el primer Heidegger. Archivos: Revista de Filosofia, 24(35), 549-579.

Di Pego, A. (2019a). En torno al pensamiento: la disputa de Hannah Arendt con Martin Heidegger. Tópicos (México), (56), 197-235. doi: 10.21555/top.v0i56.968

Di Pego, A. (2019b). Las huellas de Heidegger en La condición humana. Una disputa soterrada en torno de la praxis aristotélica. Ideas y Valores, 68(171), 185-207. doi: 10.15446/ideasyvalores.v68n171.62985

Dreyfus, H.L. (2006). Entre la 'tekné' y la técnica: el ambiguo lugar del útil en Ser y Tiempo. En I. Sabrovsky. (Ed.), La técnica en Heidegger [Vol. 2] (pp. 45-68). Santiago de Chile, Chile: Ediciones Universidad Diego Portales.

Esguerra Lozada, M. (2018). El mundo de la fenomenología de Heidegger. Una aproximación al mundo desde los Prolegómenos para una historia del concepto de tiempo. Praxis Filosófica, (46), 151-169. doi: 10.25100/pfilosofica.v0i46.6165

Feenberg, A. (2001). The Ontic and the Ontological in Heidegger's Philosophy of Technology. Inquiry, 43(4), 445-450. doi: 10.1080/002017400750051242

De Lara, F. (2014). El gesto político de las filosofías de Heidegger. Contribución a un debate actual. Veritas, (31), 73-86. doi: 10.4067/S0718-92732014000200004

Gadamer, H.-G. (2003). Heideggers 'theologische' Jugendschrift. En: G. Neumann (Ed.), Phänomenologische Interpretationen zu Aristoteles. Ausarbeitung für die Marburger und die Göttinger Fakultät (pp.76-86). Stuttgart, Alemania: Reclam.

Gethmann, C. F. (1993). Dasein: Erkennen und Handeln: Heidegger im phänomenologischen Kontext. Berlin, New York: de Gruyter. 
Gethmann, C. F. (1989). Heideggers Konzeption des Handelns in "Sein und Zeit". En A. Gethmann-Siefert y O. Pöggeler. (Eds), Heidegger und die praktische Philosophie (pp. 140 - 176). Frankfurt, Alemania: Suhrkamp.

Gethmann C. F. (2007). Vom Bewusstsein zum Handeln. Das phänomenologische Projekt und die Wende zur Sprache. Múnich, Alemania: Wilher Fink Verlag.

Heidegger, M. [SGH]. (1977). Política, Técnica y filosofía (entrevista de Der Spiegel). Revista de la Universidad Nacional, (15), 43-70.

Heidegger, M. [PS]. (1992). Platon: Sophistes (Wintersemester). Frankfurt, Alemania: Klostermann.

Heidegger, M. [ZEG]. (1994a). Debate en torno al lugar de la serenidad (de un diálogo sobre el pensamiento en un camino de campo). En M. Heidegger. Serenidad (pp. 31-92). Barcelona, España: Ediciones Del Serbal.

Heidegger, M. [G]. (1994b). Serenidad (Y. Zimmermann, trad.). Barcelona, España: Ediciones Del Serbal.

Heidegger, M. [SuZ]. (2000). Ser y tiempo (J. Gaos, trad.). Buenos Aires, Argentina: FCE.

Heidegger, M. [VA]. (2001). Conferencias y artículos. Barcelona, España: Ediciones del Serbal.

Heidegger, M. [GbaP]. (2002a). Grundbegriffe der aristotelischen Philosophie. Frankfurt, Alemania: Klostermann.

Heidegger, M. [PhIA]. (2002b). Interpretaciones fenomenológicas sobre Aristóteles. Indicación de la situación hermenéutica (Informe Natorp) (J. A. Escudero, trad.). Madrid, España: Trotta.

Heidegger, M. [EM]. (2003). Introducción a la metafisica (A. Ackermann Pilári, trad.) Barcelona, España: Gedisa.

Heidegger, M. [BP]. (2005). La idea de la filosofía y el problema de la concepción del mundo (J. A, Escudero, trad.). Barcelona, España: Herder.

Heidegger, M. [BPE]. (2006). Aportes a la filosofia. Acerca del Evento. Buenos Aires, Argentina: Biblos.

Heidegger, M. [BH]. (2007a). Carta sobre el 'humanismo'. En M. Heidegger. Hitos (pp. 259-297). Madrid, España: Alianza.

Heidegger, M. [WM]. (2007b). Hitos (H. Cortés, trads.). Madrid, España: Alianza. Heidegger, M. [OHF]. (2008a). Ontología. Hermenéutica de la facticidad. Madrid, España: Alianza.

Heidegger, M. [ID] (2008b). Identidad y diferencia (H. Cortes y A. Leyte, trad.). Barcelona, España: Anthropos.

Herrmann, F-W von. (1994). Wege ins Ereignis. Zu Heideggers "Beiträge zur Philosophie". Frankfort, Alemania: Vittorio Klostermann.

Herrmann, F-W von. (1997). Die 'Beitrāge zur Philosophie' als hermeneutischer Schlüssel zum Spātwerk Heideggers. En M. Happel, (Ed.). Heidegger neu gelesen (pp. 75-86). Wurzburgo, Alemania: Königshausen und Neumann.

Ihde, D. (1979). Technics and Praxis. Boston, USA: D. Reidel Publishing Company. Inverso, H. (2019). Fenomenología de lo inaparente. Buenos Aires, Argentina: Prometeo. 
Muñoz, A. (2010). Anotaciones sobre una posible fundamentación de la ética en Ser y Tiempo de Martin Heidegger. Praxis Filosófica, (31), 99-108. doi: 10.25100/ pfilosofica.v0i31.3429

Rentsch, T. (1989). Martin Heidegger. Das Sein und der Tod. Eine kritische Einführung. Múnich, Alemania: Piper.

Taminiaux, J. (1989). Lectures d'Ontologie Fundamentale. Grenoble, Francia: Millon.

Thanassas, P. (2012). Phronesis vs. Sophia: On Heidegger's ambivalent Aristotelianism. The Review of Metaphysics, 66(1), 31-59. doi: 10.2307/41635551

Thurnher, R. (1991). Heideggers Denken als Fundamentalethik? En R. Margreiter y K. Leidlmair. (Eds.), Heidegger: Technik, Ethik, Politik., Würzburg (pp. 133141). Wurzburgo, Alemania: Königshausen und Neumann Verlag.

Towarnick, F. y Palnier J. (1981). Conversación con Heidegger (J. Díaz Báez, trad.). Palos de la crítica, (4), 1-16.

Volpi, F. (1994). La existencia como praxis. Las raíces aristotélicas de la terminología de Ser y Tiempo. En G. Vattimo. (Comp.), Hermenéutica y racionalidad (pp. 327-383). Barcelona, España: Grupo Editorial Norma.

Volpi, F. (1996). ¿Es aún posible una ética? Heidegger y la filosofía práctica. Seminarios de filosofia, (9), 45-73.

Volpi, F. (2007). In Whose Name? Heidegger and 'Practical Philosophy'. European Journal of Political Theory, 6(1), 31-51. doi: 10.1177/1474885107070828

Volpi, F. (2012). Heidegger y Aristóteles. Ciudad de México, México: FCE.

Walton, R. (2012). El "viraje" en los "Beiträge" de M. Heidegger y en los manuscritos $\mathrm{C}$ de $\mathrm{E}$. Husserl. Investigaciones fenomenológicas: Anuario de la Sociedad Española de Fenomenología, (9), 89-115. 